\title{
Pregnancy after surgical resection of cesarean scar ectopic: A case report and literature review
}

\author{
Sasha Davidson ${ }^{1}$, Yumei Fuํㅜ and Kafui Demasio ${ }^{1}$ \\ ${ }^{1}$ Department of Obstetrics and Gynecology and Women's Health Division of Maternal Fetal Medicine Albert Einstein College of Medicine, Montefiore Medical \\ Center, Bronx, NY 10461, USA \\ ${ }^{2}$ Department of Pathology Albert Einstein College of Medicine, Montefiore Medical Center, Bronx, NY 10461, USA
}

\begin{abstract}
Background: Cesarean scar pregnancies (CSP) are associated with hemorrhage, hysterectomy and morbidly adherent placentation. Early detection is amenable to fertility preserving treatments, but a comprehensive discussion about subsequent pregnancy outcomes, especially after treatments such as wedge resection of the uterus, is lacking.
\end{abstract}

Case: A 38 year-old woman with prior cesarean section was diagnosed with CSP. After failed medical management and uterine artery embolization, she underwent laparotomy for resection of the scar. She became pregnant again, and with close pregnancy follow up, delivered at 37 weeks gestation by an uncomplicated repeat cesarean section.

Objective: This case discusses the concerns of pregnancies that follow a CSP, and reviews the literature on outcomes after available treatment modalities. We particular discuss surgical wedge resection that may be a safe and primary option for the treatment of CSP in women desiring to preserve fertility. This information is relevant when counseling women newly diagnosed with CSP.

\section{Teaching points}

- There are few reports of pregnancy following wedge resection for CSP, and the outcomes reported should not be confused with wedge resection for interstitial ectopic.

- Wedge resection of a cesarean scar pregnancy appears to be a viable option to preserve future fertility, and prevent severe morbidity but patient selection and counseling are essential.

- Elective repeat cesarean section at 37 weeks gestation appears to minimize the risk of uterine rupture and hemorrhage for women pregnant after treatment for CSP.

\section{Introduction}

There is an increased awareness of pregnancies implanted in cesarean scars that is likely due to advances in ultrasound technology, widespread use of first trimester ultrasound examination of the fetus, as well as the increase prevalence of cesarean deliveries. A cesarean scar pregnancy (CSP) is considered a rare form of an ectopic pregnancy characterized by blastocyst implantation within the endometrial defect of a prior cesarean incision. The abnormal invasion by trophoblastic cells of the myometrium, and fibrous cesarean scar, makes this type of ectopic pregnancy extremely dangerous in that if unrecognized, can result in sequelae of uterine rupture, hemorrhage, hysterectomy, and maternal death $[1,2]$. A Pregnancy that is implanted within the cesarean scar are recommended for termination due to the severity of complications and poor outcomes. Termination is usually accomplished with methotrexate [1]. However, various treatment modalities have been used either individually or in combination to appropriately treat the CSP. Treatment modalities include methotrexate (systemic or local), prophylactic uterine artery embolization followed by dilation and curettage, and rarely surgical wedge resection. There is no agreement or consensus on the best form of treatment for CSP, and the information available about subsequent pregnancy outcomes are scattered making it difficult to counsel women who are faced with this complication. We present a case describing a pregnancy course and outcome after a woman was treated for a scar ectopic pregnancy, and desired to preserve her fertility. We discuss wedge resection of the lower uterine pole and review the medical literature that discusses subsequent pregnancies after treatment of a CSP.

\section{Case}

A 38 year old female, Gravida 7 Para 1, presented to a Maternal Fetal Medicine specialist (MFM) for early prenatal care due to her prior history of a CSP.

\section{Past history}

She had 3 preterm deliveries, and one of these deliveries occurred at 26 weeks gestation by low transverse cesarean section for spontaneous preterm labor and a footling breech presentation. Eight years after that cesarean section she conceived her 6th pregnancy and was diagnosed with a CSP at 6 weeks gestation (Figure 1). The diagnosis was made by routine transvaginal ultrasound examination, the serum $\beta$ - hCG level was $70,602 \mathrm{mIU} / \mathrm{mL}$, and the patient was asymptomatic without

Correspondence to: Kafui Demasio, MD, MPH, FACOG, Montefiore Medical Center, Department of Obstetrics \& Gynecology and Women's Health 1825 Eastchester Road Bronx, NY 10461, USA; Tel: 914-260-4811; E-mail:kdemasio@ montefiore.org

Received: May 07, 2017; Accepted: May 26, 2017; Published: May 29, 2017 


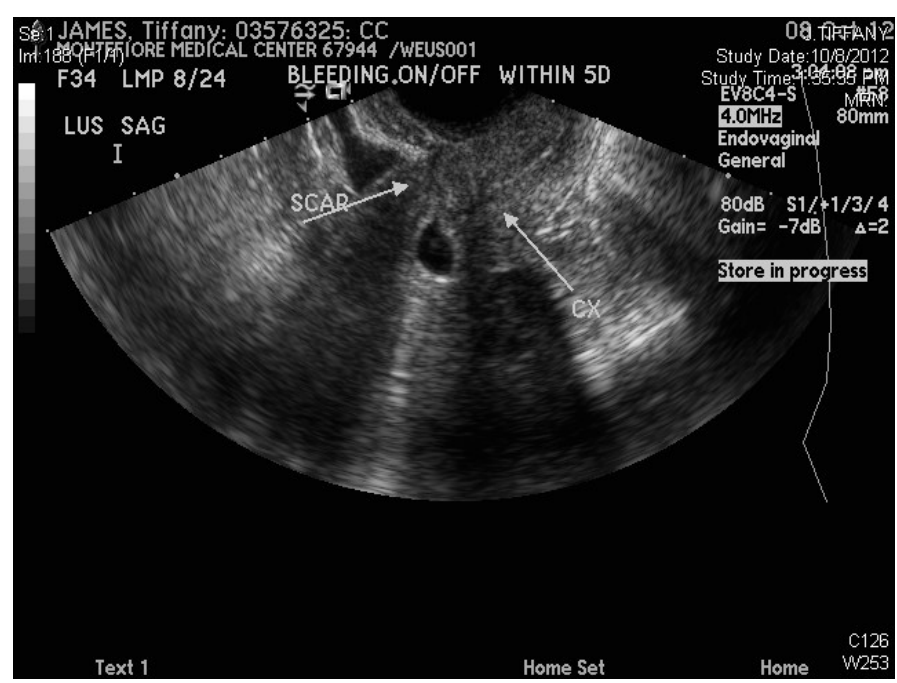

Figure 1. Transvaginal Sonogram

A sagittal view shows the gestational sac implanted at the lower pole of the uterus adjacent to the cervix (arrow). Bladder is anterior to the scar (arrow) on the uterus.

pain or vaginal bleeding. After counseling, she consented to pregnancy termination with methotrexate in order to preserve future fertility. She received a total of 3 doses ( $50 \mathrm{mg} / \mathrm{m}^{2}$ intramuscularly) and was monitored by transvaginal ultrasound examinations along with $\beta$ hCG levels. Three months passed and the $\beta$ - hCG level plateaued at $416 \mathrm{mIU} / \mathrm{mL}$. Repeat transvaginal ultrasound demonstrated persistent of the gestational sac with abnormal Doppler imaging. Because of concern for the development of uterine arteriovenous malformation, she was offered and agreed to uterine artery embolization (UAE), which was performed, and successfully stopped flow to the uterus (Figure 2). Follow up ultrasound again failed to demonstrate resolution of the cesarean scar ectopic, and the serum $\beta$ - hCG level remained at 400 $\mathrm{mIU} / \mathrm{mL}$ making this CSP intractable. At this point surgical wedge resection of the uterine scar defect was proposed and accepted by the patient. The procedure was performed through a laparotomy where a $4 \times 3 \mathrm{~cm}$ wedge was excised from the anterior lower body of the uterus and the defect closed in two layers. Pathology confirmed the presence of immature placental villi near the decidua (Figure 3).

\section{Outcome}

Two years after the wedge procedure our patient was pregnant with her $7^{\text {th }}$ pregnancy. An intrauterine gestation was confirmed, and because of the prior early premature deliveries, a Shirodkar cerclage was placed for a diagnosis of cervical insufficiency. Our patient was monitored closely throughout the pregnancy for her additional comorbidities of chronic hypertension, diabetes mellitus, and obesity. At 37 weeks gestation a healthy, but small female infant weighing 4 pounds 6 ounces was delivered by repeat cesarean section. The delivery was uncomplicated, without evidence of uterine dehiscence. The estimated blood loss for the procedure was $500 \mathrm{cc}$.

\section{Discussion}

Cesarean scar implantation is rare with an incidence of $1: 3000^{3}$ to $1: 2200^{1}$ pregnancies in the general population. A higher incidence of 1 in 531 women is reported for women with a history of at least one cesarean delivery [3]. Cesarean scar implantation represents $4-6 \%$ of all ectopic pregnancies in these populations. Presumably correlated with the increase in cesarean deliveries, a prior cesarean section is a prerequisite for scar ectopic pregnancy, therefore the increasing cesarean rate is likely to yield more women diagnosed with CSP. The pathogenesis of a CSP is thought to involve implantation through a "microscopic dehiscent tract" [4], or a defect in the scar from aberrant full-thickness healing at the time of the original cesarean section [5]. Factors thought to predispose to poor myometrial healing during the original cesarean section are preterm gestation, breech presentation, cesarean following failure to progress in early labor (where there is underdevelopment of the lower uterine segment), and closure of the hysterotomy with monolayer non-inverting running sutures [5]. In -vitro fertilization has also been implicated as another risk factor leading to a deficient myometrium [2]. Woman diagnosed with CSP should be informed about treatment options and how treatment impacts future reproduction. In particular, wedge resection of the scar may be the preferred treatment option for women who desire pregnancy after the detection of a CSP.

Wedge resection procedures have been described primarily in the setting of interstitial or cornual ectopic pregnancies [6]. The anatomical location of an interstitial ectopic is different from a CSP in that implantation occurs at the junction of the fallopian tube and
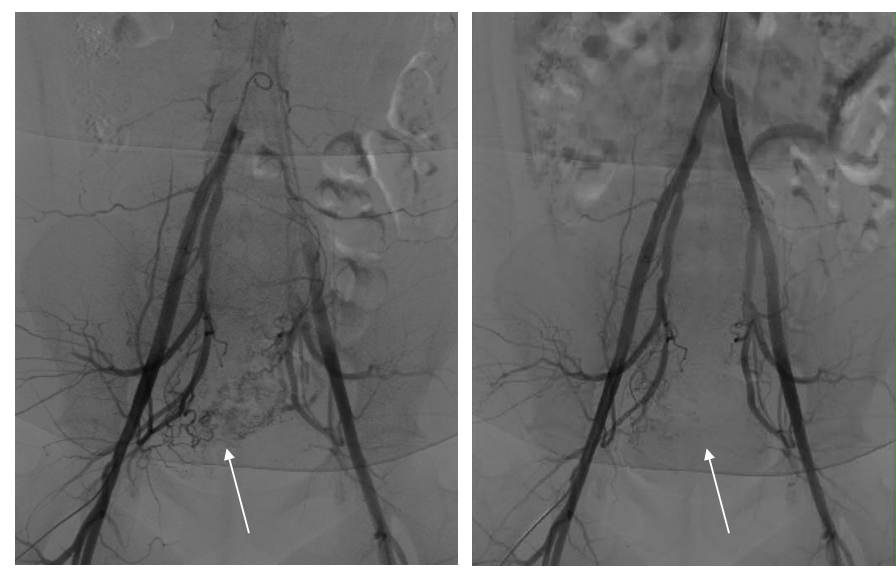

Figure 2. Pre and Post-Embolization Angiogram.

(A) A hypervascular blush is seen involving the lower aspect of the uterus. (B) After occlusion of both uterine arteries there is devascularization and resolution of this hypervascular area.

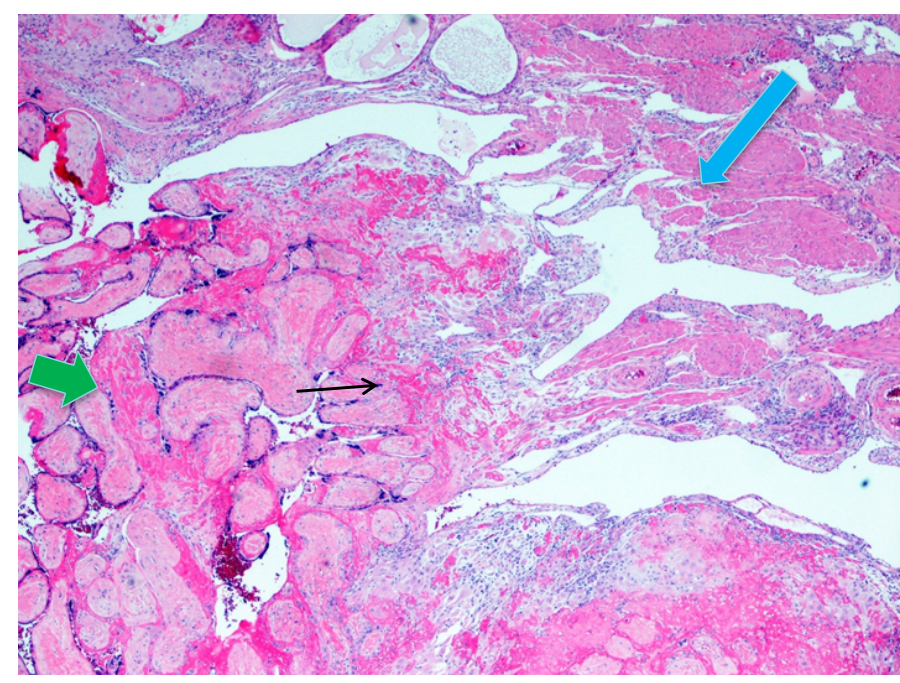

Figure 3. Wedge Resection Specimen

H\&E stain (medium power) shows immature chorionic villi (short green arrow) adjacent to the decidua (black arrow). Smooth muscle bundles of uterine myometrium (blue arrow) are located to the right of decidua. 
uterine fundus whereas a CSP implants within the fibrotic scar of the body of the uterus. Pregnancy after wedge resection of an interstitial ectopic has the significant risk of uterine rupture but wedge resection of a CSP has a more favorable outcome.

There are two proposed types of CSP: the first involves implantation of the gestational sac on the scar, with progression toward either the cervicoisthmic space or toward the uterine cavity. The second proposed type is characterized by deep implantation into the myometrial defect with the sac continuously remaining outside the uterine cavity[6]. We suggest that a CSP resulting from the latter type is more likely to fail methotrexate treatment and less accessible to devascularization from UAE. A CSP of the second type may be more amenable to treatment by removal of the fibrotic scar and pregnancy tissue by wedge resection. Although methotrexate has been used extensively in the management of tubal and cervical ectopic pregnancies, the route, dosing and efficacy in treating a CSP has yet to be established. Methotrexate has variable success rates, and is associated with reports of failure requiring additional intervention. Sadeghi et al reviewed the medical literature and reported on 268 cases of CSP, 27 were treated with methotrexate as an initial therapy and 59\% of those cases required additional intervention [5]. Although factors that lead to methotrexate failure are not clearly established for CSP, a high failure rate of methotrexate has been shown with cervical ectopic pregnancies that are $\geq 9$ weeks gestation, a fetal pole $\geq 10 \mathrm{~mm}$, the presence of cardiac activity, and $\beta$-hCG concentration $\geq 10,000$ IU per liter.

Pregnancy outcomes reported after surgical wedge resection are largely from the treatment of interstitial ectopic pregnancies. Information is lacking about subsequent pregnancies following wedge resection of a CSP. We sought to summarize the outcome of future pregnancies after wedge resection of the lower pole of the uterus. We searched the medical literature for articles published in English that reported the outcome of subsequent pregnancies following wedge resection of CSP. We found only 4 other cases describing a subsequent pregnancy after some form of surgical resection of the lower pole of the uterus (Neiger 1998, Vial 2000, Nagi 2006, Siedhoff 2015). Subsequent pregnancies after surgical resection were not reported in the series by $\mathrm{Seow}^{2}$ or Maymon ${ }^{3}$, and therefore were not included. With the addition of this report, 5 cases of pregnancy outcomes after resection of the lower pole of the uterus are detailed in the medical literature.

Case \# 1 was a 28 year old gravida 3 para 2 , who underwent resection of the gestational sac and closure of the scar at 6 weeks gestation [6]. This patient conceived 2 months later and had an uneventful pregnancy course ending with a repeat cesarean section at term. Case \#2 was a 39 year old with 2 prior cesarean sections who first presented with a 12 week CSP treated with laparoscopy followed by suction evacuation [1]. She had two subsequent CSPs at 7 and 4 weeks gestation treated with suction curettage. A laparotomy was performed 3 months after the third CSP with excision and repair of the uterine scar followed by a 3-layer closer. This patient went on to have 2 normally implanted pregnancies, however had 2 first trimester recurrent losses. Case \#3 describes a 38 year old gravida 6 para 2 with two prior cesarean sections and a third IVF pregnancy complicated by CSP [7]. She underwent a robotic assisted laparoscopic repair of the uterine defect. The scar was resected and a 2 layer closure was performed. She conceived spontaneously 18 months later and delivered at 37 weeks by scheduled repeat cesarean section. Case \# 4 is a 21 year old gravida 4 para 2 with a history of two prior cesarean deliveries; first for breech and the second as an elective repeat [8]. She was found to have a CSP and underwent laparotomy with wedge resection of the ectopic pregnancy. The uterine defect was closed in two layers. She became pregnant 6 months later with dichorionic diamniotic twins and had an uneventful pregnancy. She delivered by repeat cesarean section at 37 weeks gestation. As in our case, there was no evidence of uterine dehiscence in any of the cases.

None of the women treated by lower uterine pole wedge resection who became pregnant experienced uterine rupture, hemorrhage or adverse outcome. We believe that the common factors seen in all the cases, which are restoring the integrity of the endometrial cavity by scar removal, multilayer repair of the uterine defect, and the elective repeat cesarean sections performed at 37 weeks of gestation prior to the onset of labor, led to the satisfactory outcomes. Four of the five women treated became pregnant and had term deliveries. The newborn for our patient was small for gestational age for unclear reasons. This finding may have been constitutional, due to maternal co-morbidity of hypertension, or vascular compromise of the uterus from previous UAE that was performed in attempting to treat the prior CSP.

Although several fertility preserving treatment modalities are described for CSP, the true measure of success is to provide a safe subsequent pregnancy. Reports of subsequent pregnancy outcomes following treatment for a CSP are necessary to establish the true benefits and risks of fertility preserving treatments. Our review of the literature for subsequent pregnancy outcomes following a CSP found very few articles that report subsequent outcome. We found that women who received methotrexate treatment for a CSP are able to become pregnant, with a risk of recurrent CSP between $20-25 \%$ $[1,3]$. Dilation and curettage to remove CSP is consistently associated with hemorrhage and the potential for hysterectomy. Uterine rupture, hemorrhage resulting in hysterectomy, and deaths have been reported in unrecognized and expectantly managed CSP [2]. Uterine artery embolization after failed response to methotrexate, has raised concern of its effect on the subsequent integrity of the uterus because the devascularization of the myometrium after UAE may predispose to an increase risk of intrauterine growth restriction or postpartum hemorrhage. We conclude, as was previously suggested [6], that the best definitive treatment and chance for successful future pregnancy may be complete surgical removal of the cesarean scar pregnancy. Additionally, multi-layered closure to restore the integrity of the endometrium and delivery at 37 weeks gestation appears to avoid the risk of adverse pregnancy outcome.

\section{References}

1. Nagi BJ, Helmy S, Ofili-Yebovi S, Yazbek J, Sawyer E et al. (2007) Reproductive outcomes of women with a previous history of cesarean scar ectopic pregnancies. Human Reproduction 22: 2012-15.[Crossref]

2. Seow KM, Hwang JL, Tsai YL, Huang LW, Lin YH et al. (2004) Subsequent pregnancy outcome after conservative treatment of a previous cesarean scar pregnancy. Acta Obstet Gynecol Scand 83: 1167-1172.[Crossref]

3. Maymon R, Svirsky R, Smorgick N, Mendlovic S, Halperin R et al. (2011) Fertility Performance and Obstetric Outcomes among women with previous cesarean scar pregnancy. J Ultrasound Med 30: 1179-84.[Crossref]

4. Godin PA, Bassil S, Donnez J (1997) An ectopic pregnancy developing in a cesarean section scar. Fertility and Sterility 67: 398-400.

5. Sadeghi H, Rutherford T, Rackow BW, Campbell KH, Duzyj CM et al. (2010) Cesarean scar ectopic pregnancy: Case series and review of the literature. Am Journal Perinatol 27: 111-120.[Crossref]

6. Vial Y, Petignat P, Hohlfeld P (2000) Pregnancy in a cesarean scar. Ultrasound Obstet Gynecol 16:592-3.

7. Siedhoff MT, Schiff LD, Moulder JK, Toubia T, Ivester T (2015) Robotic-assisted laparoscopic removal of cesarean scar ectopic and hysterotomy revision. Am J Obstet Gynecol 212: 681.e1-4.[Crossref]

8. Neiger R, Weldon K, Means N (1998) Intramural pregnancy in a cesarean section scar Journal of Reproductive Medicine 43:999-1001.[Crossref]

Copyright: (C)2017 Davidson S. This is an open-access article distributed under the terms of the Creative Commons Attribution License, which permits unrestricted use, distribution, and reproduction in any medium, provided the original author and source are credited. 